\title{
Endometrial carcinoma: immunohistochemically detected proliferation index is a prognosticator of long term outcome
}

\author{
A M Gassel, J Backe, S Krebs, S Schön, H Caffier H K Müller-Hermelink
}

\begin{abstract}
Aim-To test which immunohistochemically detected tumour parameters are predictive of outcome in endometrial carcinoma.

Methods-A retrospective study of 300 patients diagnosed with endometrial carcinoma between 1980 and 1985, ensuring a follow up of at least 10 years. Paraffin wax embedded tissues from 236 patients with endometrial carcinoma were evaluated in terms of histological tumour type and grade, stage of disease, and certain immunohistochemical biological parameters. These parameters included the expression of oestrogen and progesterone receptors, the expression of p53 protein, the expression of the c-erbB-2 oncoprotein, and the expression of protease cathepsin $D$, together with the rate of cell proliferation. Results-Using univariate analysis, the following parameters correlated significantly with adjusted survival: histological type $(p=0.025)$, grade $(p=0.00003)$, FIGO stage $(p<0.00001)$, proliferation rate $(p=0.00002)$, oestrogen receptor expression $(p=0.007)$, progesterone receptor expression $(p=0.0092)$, and $p 53$ expression $(p=0.00028)$. These parameters also correlated significantly with both disease free and overall survival. There was a weak correlation of cathepsin $D$ expression with survival, but no correlation of c-erbB-2 expression with survival. Using multivariate analysis, only FIGO stage $(p=0.0021)$, histological grade $(p=0.005)$, and proliferation rate $(p=0.0007)$ remained statistically significant prognosticators of adjusted survival as well as of disease free and overall survival.
\end{abstract}

Conclusions-In addition to conventional histological parameters, the immunohistochemical determination of proliferative activity could contribute to the identification of a high risk subgroup of endometrial carcinomas. The other parameters tested were not of significant additional predictive value.

(F Clin Pathol 1998;51:25-29)

Keywords: endometrial carcinoma; prognosticators; proliferation rate

Endometrial carcinoma is one of the most frequent and also one of the most curable of cancers affecting women ${ }^{1}$ with 10 year survival rates of up to $97 \%$ in low risk groups. ${ }^{2}$
However, if recurrence occurs, the survival rate drops significantly. To identify patients with a high risk of recurrence, and to prevent uneccessary treatment in patients with low risk disease, prognostic factors need to be defined clearly. These include stage, ${ }^{3}$ grade, histological type, patient's age,${ }^{4}$ tumour size,${ }^{5}$ hormone receptor status, ${ }^{6}$ and oncogene expression. ${ }^{78}$ When performing routine surgical pathology, it is difficult to test for all of these factors. This study aimed at identifying factors associated independently with survival rates. Hopefully, such factors will be useful both in the individualisation of treatments and to help minimise diagnostic expenses.

\section{Methods}

PATIENTS

Three hundred patients with a diagnosis of endometrial carcinoma from 1980 to 1985 were studied retrospectively, ensuring a follow up of at least 10 years. Data were collected from the clinic of obstetrics and gynaecology, University of Würzburg and from office records of the patients' community. The mean age was 67 years (41-94 years). One hundred and eighty three patients $(61 \%)$ underwent primary surgical therapy. One hundred and seventeen patients $(38 \%)$ received primary pelvic irradiation owing to their reduced health status. Additional postoperative radiation was performed on $46 \%$ of the operated patients. Indications were stage 1 tumours infiltrating more than $3 \mathrm{~mm}$ of the myometrium, as well as stage 2 and stage 3 disease. Most of the patients had the following risk factors known to be associated with endometrial carcinoma: overweight (up to $30 \%$ overweight, $38 \%$; up to $60 \%$ overweight, $24 \%$; up to $90 \%$ overweight, 5\%), hypertonus $(58 \%)$, and diabetes $(29 \%)$.

TYPING AND GRADING

All cases were reclassified and histological typing and grading was performed according to the WHO classification of endometrial carcinoma from 1994 and the FIGO criteria. ${ }^{9}$

\section{STAGING}

All cases were restaged. Patients who underwent primary surgery were staged according to the FIGO classification of 1988 . The patients receiving primary radiation therapy were staged clinically, using the adopted FIGO classification from 1971. 
IMMUNOHISTOCHEMISTRY

Slides from 236 cases were reviewed and analysed by immunohistochemistry. All immunohistochemical stainings were evaluated by two independent observers blinded to the clinical data.

\section{Proliferation}

The growth fraction was determined by immunohistochemical detection of proliferating cells, using the monoclonal antibody $\mathrm{Ki}-\mathrm{S} 1$. This antibody is directed against topoisomerase II- $\alpha^{10}$ and reacts with all phases of the cell cycle except $\mathrm{G}_{0}$. It can be applied to paraffin wax embedded sections after microwave treatment as described by Kreipe et al using a peroxidase antiperoxidase technique and rabbit antimouse antibody. The percentage of positively stained tumour cell nuclei was counted in five areas of the slide with maximum staining intensity and the mean value was taken. According to the percentage of proliferating cells the tumours were classified as low ( $<20 \%$ proliferation), medium (21-50\% proliferation), and high proliferating (51-100\% proliferation).

\section{Hormone receptor status}

Oestrogen and progesterone receptor expression was determined immunohistochemically using both oestrogen and progesterone receptor specific antibodies and staining kits from Abbott (Wiesbaden, Germany). The scoring system of Remmele and Stegner ${ }^{11}$ was used: an immunoreactive score was derived by multiplying the staining intensity (negative $=0$, mild $=1$, moderate $=2$, strong $=3$ ) with the percentage of positive tumour cell nuclei ( $0=$ none, $1=<10 \%, 2=10-50 \%, 3=51-$ $80 \%, 4=>80 \%)$. In accordance with Segreti et $a l,{ }^{12}$ an immunoreactive score of 2 and higher was considered to be positive.

\section{p53 expression}

Expression of the tumour suppressor gene product was determined with the help of the p53 antibody DO-1 (Immunotech, Hamburg, Germany). This antibody reacts with wild-type and mutant oncoprotein in formalin fixed, paraffin wax embedded material. It was applied undiluted to microwave pretreated slides as described previously by Pezzella et al. ${ }^{13}$ The positive tumour cells were identified using the immunoreactive score described for hormone receptors.

c-erbB-2 expression

Expression of the oncoprotein c-erbB-2 (HER2/neu) was determined by immunohistochemistry using the monoclonal antibody CB11 (BioGenex, San Ramon, California, USA). This antibody was applied according to the manufacturer's protocol. Percentage and intensity of tumour cell membrane activity was determined with the help of the abovementioned scoring system.

Cathepsin D expression

Expression of the lysosomal proteinase cathepsin D was determined immunohistochemically by evaluating the number of reactive tumour cells and the staining intensity of the monoclonal antibody Ab-1 (Oncogene Science, Cambridge, Massachusetts, USA). The antibody was applied as suggested by the manufacturer and visualised with an immunoperoxidase technique, as described above. In accordance with previously published data from breast cancers ${ }^{14}$ we used the same scoring system as for hormone receptors (see above). Immunoreactive stromal cells were not evaluated.

\section{STATISTICS}

Statistical analyses were performed using the software SPSS (Statistical Package for the Social Sciences, Munich, Germany) for Windows. The Spearman rank test was used to correlate oestrogen and progesterone receptor expression. Evaluation of follow up data was based on the log rank test and was visualised by Kaplan-Meier curves. For multivariate analysis, the Cox proportional hazards model was used to assess the relative strength of multiple covariates. Differences of $p \leqslant 0.05$ were considered to be statistically significant.

\section{Results}

During the follow up period (0-15 years) 120 patients died because of malignancy and 84 patients died owing to intercurrent disease. The outcome appeared to be treatment related, with a longer survival in patients who had been operated on compared to those who had not. A correction was made for this difference in the multivariate analysis.

TYPING

Two hundred and fourteen tumours (91\%) were adenocarcinomas with endometroid differentiation, including 36 adenoacanthomas and 12 adenosquamous carcinomas. Only 9\% of the tumours displayed non-endometroid features with seven clear cell carcinomas, eight serous carcinomas, and seven undifferentiated carcinomas ( $3 \%$ each). Histological type had a significant influence on adjusted survival $(p=0.025)$ and a slight influence on overall survival $(\mathrm{p}=0.072)$ in univariate analysis. Disease free survival was not associated with histological type $(p=0.6)$. Endometroid carcinomas including adenoacanthoma had the best prognosis, followed by adenosquamous carcinoma then serous, clear cell, and undifferentiated carcinomas. Owing to the low frequency of non-endometroid tumours, the latter three were analysed together. However, this factor was not an independent prognosticator when multivariate analysis was used $(\mathrm{p}=0.11)$.

\section{GRADING}

One hundred and seventy one tumours (72\%) were either well differentiated or moderately differentiated (grade 1 and 2) and 65 tumours $(27.5 \%)$ were poorly differentiated (grade 3 ). No significant difference for survival was found between grade 1 and 2 tumours, but the adjusted survival was significantly worse for grade 3 tumours $(p=0.00003)$. This correlation was also significant for overall survival $(p=0.002)$ and disease free survival 


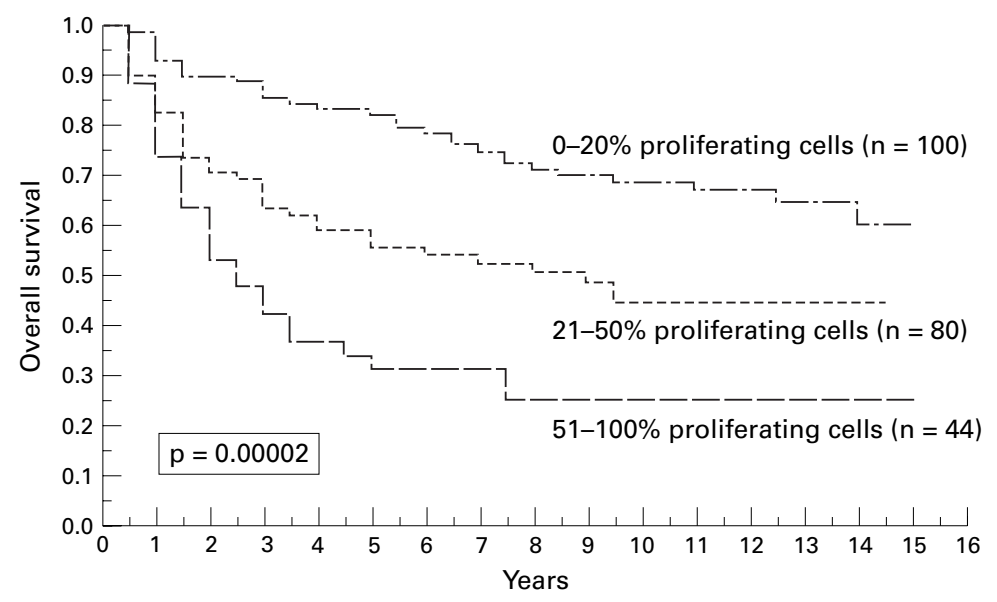

Figure 1 Kaplan-Meier curve for overall survival in patients with endometrial carcinoma, classified according to the percentage of Ki-S1 positive proliferating tumour cells.

$(p=0.022)$. It remained significant when multivariate analysis was used $(\mathrm{p}=0.005)$.

STAGING

One hundred and fifty one patients had stage 1 disease, 88 patients had stage 2 disease, and 29 patients had advanced tumour stages $3 \mathrm{a}$ and $\mathrm{b}$. Six cases consisted of carcinomas in situ. Stage of disease and adjusted survival were significantly correlated $(p<0.00001)$. The same holds true for overall survival $(\mathrm{p}<0.00001)$ and disease free survival $(p=0.022)$. In stage 1 tumours, there was a significantly longer survival for patients with infiltration of less than one half of the myometrium compared with patients who had greater than one half infiltration $(p=0.03)$. The borderline for postoperative radiation was $3 \mathrm{~mm}$ of infiltration. There was a slight tendency towards worse survival and earlier disease recurrence in patients with more than $3 \mathrm{~mm}$ infiltration, although this was not significant.

PROLIFERATION

The rate of tumour cell proliferation was determined in 224 specimens. One hundred cases $(45 \%)$ had a proliferation rate of $20 \%, 80$ cases $(35 \%)$ displayed a proliferation rate ranging between $21 \%$ and $50 \%$, and 44 cases $(20 \%)$ had a proliferation rate of between $51 \%$ and $100 \%$. The proliferation rate correlated significantly with overall survival $(\mathrm{p}=0.00002$; fig 1). A significant correlation with recurrence free survival $(\mathrm{p}=0.018)$ and adjusted survival $(\mathrm{p}=0.0012)$ was also found. This proliferation rate remained an independent prognostic factor when multivariate analysis was used $(p=0.0007)$. Proliferation correlated significantly with histological grade, but only in the combination of grade 1 and $2(p<0.00001)$. There was a strong correlation between p53 overexpression and highly proliferating tumours ( $\mathrm{p}=0.00024$; fig 2 ).

\section{HORMONE RECEPTORS}

One hundred and forty seven specimens were eligible for oestrogen receptor analysis and 227 cases for progesterone receptor analysis. This disparity is due to the high number of cases that were negative for oestrogen receptor staining of their myometrial cell nuclei, which served as an internal control. These cases were older than 10 years, suggesting that the negative staining results were probably due to breakdown of the antigen. Sixty of 147 cases $(41 \%)$ were oestrogen receptor negative and 87 cases $(59 \%)$ were positive. In progesterone receptor immunohistochemistry, 120 of 227 cases (53\%) were negative, 107 of 227 cases (47\%) displayed positive reactivity. Using univariate analysis, both oestrogen and progesterone receptor expression correlated significantly with adjusted survival $(\mathrm{p}=0.007$ and 0.0092 for oestrogen and progesterone receptors, respectively). Moreover, we found a significant association with overall survival $(\mathrm{p}=0.0099$ and 0.05 for oestrogen and progesterone receptors, respectively). Using multivariate analysis, however, this effect was no longer significant $(\mathrm{p}=0.32$ and 0.46 for oestrogen and progesterone receptors, respectively) and we could not find a significant association with recurrence free survival.

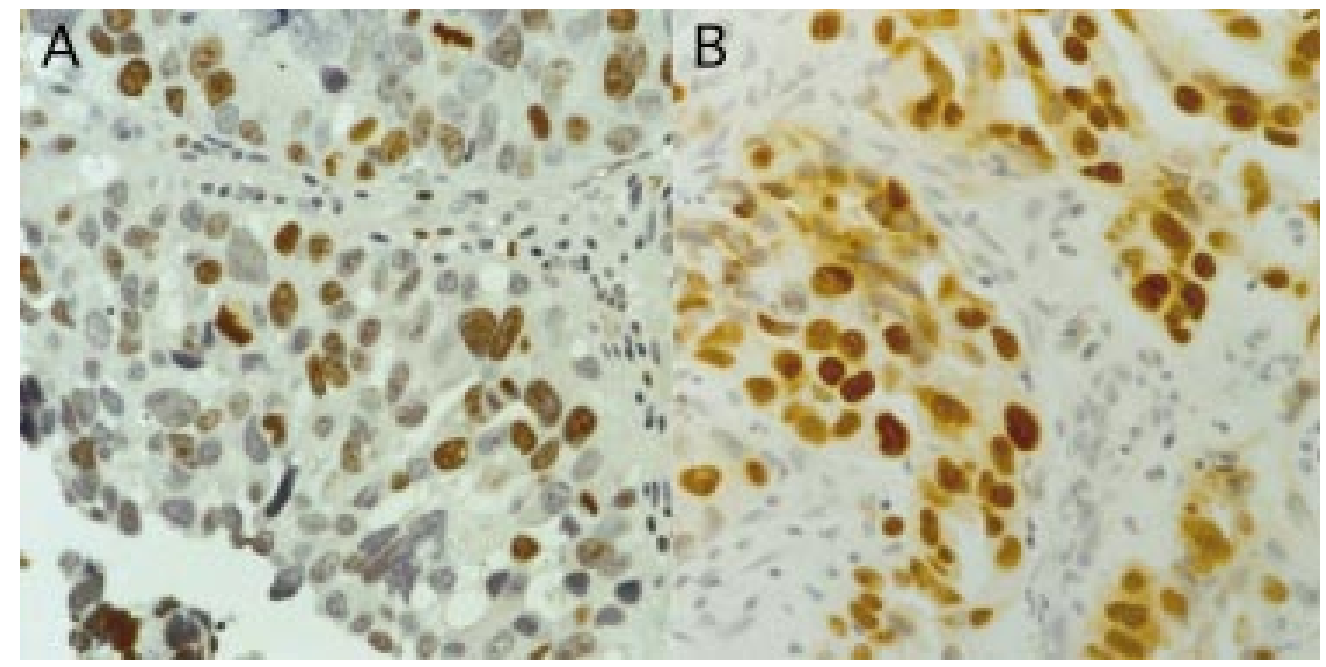

Figure 2 (A) Highly proliferating endometrial carcinoma containing up to 80\% Ki-S1 positive tumour cell nuclei. (B) The same tumour exhibits a strong overexpression of the p53 protein, with $>70 \%$ of tumour cells strongly positive for the monoclonal antibody DO-1. (Immunoperoxidase stained; original magnification $\times 450$.) 
Table 1 Multivariate analysis for adjusted survival in patients with endometrial carcinomas

\begin{tabular}{lll}
\hline & \multicolumn{2}{l}{ Adjusted survival } \\
\cline { 2 - 3 } Variable & Number & p value \\
\hline Proliferation rate (Ki-S1) & 224 & 0.0007 \\
FIGO stage & 236 & 0.0021 \\
Grade & 236 & 0.005 \\
Type & 236 & 0.11 \\
c-erbB-2 expression & 222 & 0.12 \\
Cathepsin D expression & 225 & 0.26 \\
p53 expression & 202 & 0.27 \\
Oestrogen receptor concentration & 147 & 0.32 \\
Progesterone receptor & & \\
$\quad$ concentration & 227 & 0.46 \\
\hline
\end{tabular}

P53 EXPRESSION

Most of the tumours eligible for $\mathrm{p} 53$ expression studies showed no overexpression of the oncoprotein. One hundred and thirty nine of 202 cases $(69 \%)$ displayed no positive reaction, whereas 63 tumours (31\%) overexpressed p53 (with an immunoreactive score $\geqslant 2$ ). The level of protein expression was significantly correlated with adjusted survival $(\mathrm{p}=0.00028)$, as well as with overall survival $(p=0.003)$ and recurrence free survival $(\mathrm{p}=0.04)$. Using multivariate analysis, however, this effect did not remain significant $(p=0.27)$ and was replaced by other parameters (table 1 ). Correlation of p53 with the proliferative status of the tumour was highly significant (see above). p53 overexpression was increased in tumours with low differentiation $(\mathrm{p}=0.0001)$ and in tumours with non-endometroid histology $(\mathrm{p}=0.0069)$.

c-erbB-2 EXPRESSION

Two hundred and twenty two tumours were eligible for c-erbB-2 expression. One hundred and nine of $222(49 \%)$ displayed no immunohistochemical reactivity and 113 of $222(51 \%)$ were reactive for this oncoprotein. We found a slight tendency towards longer adjusted survival in low expressing tumours $(\mathrm{p}=0.18)$. Correlations with overall survival or recurrence free survival could not be detected.

CATHEPSIN D EXPRESSION

Two hundred and twenty five cases were evaluated for cathepsin D expression. There was little intratumoural variation of staining intensity. We could not find more intensive staining in the infiltrating borderline of the tumour. In cases with additional endometrial hyperplasia, the hyperplastic glands showed a weak positivity, whereas normal endometrium was nonreactive. One hundred and twenty of 225 tumours $(53 \%)$ showed low level expression of cathepsin D and 105 (47\%) were strongly positive. There was a significant correlation between overall survival and expression of this protein in the univariate analysis $(p=0.048)$. However, neither tumour stage, depth of infiltration, adjusted survival, nor recurrence free survival were associated with cathepsin D expression.

Using multivariate analysis (table 1), the following independent factors remained significantly correlated with survival: FIGO stage $(p=0.0021)$, histological grade $(p=0.005)$, and proliferation rate $(\mathrm{p}=0.0007)$.

\section{Discussion}

Tumour type, grade, and stage are established parameters for use in the prognostication of the outcome of endometrial carcinoma. The distribution and prognosis of histological subtypes in this study are comparable to those of other investigators. ${ }^{15}{ }^{16}$ It is likely that the increasing frequency of tumours with non-endometroid histology in the last decade ${ }^{17}$ reflects an altered postmenopausal hormone substitution; this does not seem to have had any effect on the cases examined in this study. In addition to conventional histological criteria, several tumour biological parameters were studied for their prognostic value; using univariate analysis, the immunohistochemically detected content of steroid hormone receptors was found to correlate significantly with survival and tumour grade. These results agree with those of Segreti et al. ${ }^{12}$ Nonetheless, unlike the results for biochemical investigations of steroid hormone receptors, this effect was not significant when multivariate analysis was applied. ${ }^{6}$ However, Maentausta et al found a good correlation between biochemical and immunohistochemical results, ${ }^{18}$ suggesting that differences could be a result of discrepancies in the follow up periods and the different scoring systems used. The generation of monoclonal antibodies directed towards proliferation associated antigens, such as $\mathrm{Ki}-67,{ }^{19}$ has enabled prospective $^{2021}$ and retrospective studies ${ }^{22}$ to be carried out. Investigating 224 cases, we found a highly significant correlation between the percentage of proliferating tumour cells and survival. This correlation is independent from the other factors associated with high proliferation rate, such as high grade and tumour stage, and it remains significant when multivariate Cox regression analysis is applied. This is in keeping with studies by Garzetti et $a l^{23}$ and Geisler et $a l,{ }^{24}$ who found a significant correlation of expression of proliferation associated antigens (proliferating cell nuclear antigen (PCNA) and MIB-1) with recurrence free survival. Contrasting results were found by Hamel et al using computerised image analysis of PCNA immunostained cells. ${ }^{25}$ No correlation of stained nuclear area with recurrence was found. This result is surprising and might be a result of technical differences in the evaluation of positive cancer cells. In the last five years, a number of studies on the prognostic value of the tumour suppressor gene product p53 have been performed. ${ }^{26}{ }^{27}$ p53 overexpression has been described in 10-15\% of early cancers and up to $50 \%$ of advanced cancers ${ }^{28}$; in addition, it is frequently associated with point mutations and loss of heterozygosity. ${ }^{29}$ Correlation of overexpression with both the spread of disease $^{30}$ and survival has been found. ${ }^{31}$ Our data are consistent with those of previous studies $^{32}$ that found a correlation of p53 overexpression with tumour grade and stage. In addition, we found p53 expression to be a prognosticator of long term survival, with a strong correlation of low expression and better outcome. However, this correlation does not remain significant in a multivariate analysis. Production of cathepsin D has been found to 
be correlated both with tumour invasiveness and stage of endometrial carcinomas, ${ }^{33}$ as well as with histological subtype. ${ }^{34}$ We could confirm the predictive value of cathepsin D only with regard to overall but not adjusted survival, which is comparable to the situation in breast cancer. ${ }^{14}$ c-erbB-2 protein expression is a matter of conflicting data: whereas some authors describe a significant correlation with disease stage $^{28}$ and worse prognosis, ${ }^{35}$ other authors do not. ${ }^{36}$ In our group of tumours, there was no significant relation between c-erbB-2 overexpression and tumour stage or survival. Glandular cells in endometrial hyperplasia also displayed expression of the c-erbB-2 protein. ${ }^{38}$ This early expression is maintained throughout the course of the disease, making its prognostic value questionable. In conclusion, our results confirm the prognostic value of conventional histopathological parameters, such as histological grade and stage, and in addition they support the value of immunohistochemically determined proliferative activity in the identification of high risk patients with endometrial carcinoma.

1 Creasman WT. Adenocarcinoma of the uterine corpus. Curr Opin Obstet Gynecol 1992;5:80-3.

2 Kadar N, Malfetano JH, Homesley HD. Determinants of survival of surgically staged patients with endometrial carcinoma histologically confined to the uterus: implications for therapy. Obstet Gynecol 1992;80:655-9.

3 Wolfson AH, Sightler SE, Markoe AM, et al. The prognostic significance of surgical staging for carcinoma of the endometrium. Gynecol Oncol 1992;45:142-6.

4 Creasman WT, Morrow CP, Bundy L, et al. Surgical pathological spread patterns of endometrial cancer. Cancer 1987; 60:2035-41.

5 Schink JC, Rademaker AW, Miller DS, et al. Tumour size in endometrial cancer. Cancer 1991;67:2791-4.

6 Creasman WT. Prognostic significance of hormone receptors in endometrial cancers. Cancer 1993;71:1467-70.

7 Khalifa MA, Mannel RS, Haraway SD, et al. Expression of EGFR, HER-2/neu, p53, and PCNA in endometrioid, EGFR, HER-2/neu, p53, and PCNA in endometrioid, serous papillary and clear cell end
mas. Gynecol Oncol 1994;53:84-92.

8 Pisani AL, Barbuto DA, Chen D, et al. HER-2/neu, p53, and DNA analyses as prognosticators for survival in endometrial carcinoma. Obstet Gynecol 1995;85:729-34.

9 Creasman, WT. Announcement, FIGO stages: 1988 revision. Gynecol Oncol 1989;35:125-7.

10 Kreipe $\mathrm{H}-\mathrm{H}$, Alm P, Olsson $\mathrm{H}$, et al. Prognostic significance of a formalin resistant nuclear proliferation antigen in mammary carcinomas as determined by the monoclonal antibody Ki-S1. Am $\mathcal{F}$ Pathol 1993;142:651-7.

11 Remmele W, Stegner HE. Vorschlag zur einheitlichen definition eines immunreaktiven score (IRS) für den immunhistochemischen östrogenrezeptor nachweis im mammakarzinomgewebe. Pathologe 1987;8:138-40.

12 Segreti EM, Novotny DB, Soper JT, et al. Endometrial cancer: histologic correlates of immunohistochemical cancer: histologic correlates of immunohistochemical localization of progester

13 Pezzella F, Micklem K, Turley H, et al. Antibody for detecting $\mathrm{p} 53$ protein by immunohistochemistry in normal tissues. 7 Clin Pathol 1994:47:592-6.

14 Göhring U-J, Scharl A, Thelen U, et al. Prognostic value of cathepsin D in breast cancer: comparison of immunohistochemical and immunoradiometric detection methods. $\mathcal{F}$ Clin Pathol 1996;49:57-64.

15 Christopherson WM, Connelly PJ, Alberhasky RC. Carcinoma of the endometrium. in patients with favorable subtypes and stage I disease. Cancer 1983;51:1705-10.

16 Kurman RJ, Norris HJ. Endometrial carcinomas. In: Kurman RJ, ed. Blaustein's pathology of the female genital tract. New York: Springer, 1994:338-72.

17 Dallenbach F, Stein H, Dallenbach FD. Proliferationsmarker bei endometriumkarzinomen verschiedener histogenese: Vergleich immunhistologischer untersuchungen an kryostat-und paraffinschnitten. Verh Dtsch Ges Path 1991;75:375.

18 Maentausta O, Boman $\mathrm{K}$, Isomaa V, et al. Immunohistochemical study of the human $17 \beta$-hydroxysteroid dehydrogenase and steroid receptors in endometrial adenocarcinoma. Cancer 1992;70:1551-5

19 Gerdes J, Lemke H, Baisch H, et al. Cell cycle analysis of a cell-proliferation associated human nuclear antigen defined by the monoclonal antibody Ki-67. F Immunol 1984;133: $1710-15$.

20 Yabushita $\mathrm{H}$, Masuda T, Sawaguchi K, et al. Growth potential of endometrial cancers assessed by a $\mathrm{Ki}-67 \mathrm{Ag} / \mathrm{DNA}$ dual-color flow-cytometric assay. Gynecol Oncol 1992;443: 263-7.

21 Nielsen AL, Nyholm CJ. Proliferative activity as revealed by $\mathrm{Ki}-67$ in uterine adenocarcinoma of endometroid type: comparison of tumours from patients with and without previous estrogen therapy. F Pathol 1993;171:199-205.

22 Dallenbach-Hellweg G, Lang-Averous G, Hahn U. The value of immunohistochemistry in the differential diagnosis of endometrial carcinomas. APMIS 1991;23(suppl):91-9.

23 Garzetti GG, Ciavattani A, Goteri G, et al. Proliferating cell nuclear antigen in endometrial carcinoma: pretreatment identification of high-risk patients. Gynecol Oncol 1996;61: $16-21$.

24 Geisler JP, Wiemann MC, Zhou Z, et al. Proliferation index determined by MIB-1 and recurrence in endometrial cancer. Gynecol Oncol 1996;61:373-7.

25 Hamel NW, Sebo TJ, Wilson TO, et al. Prognostic value of p53 and proliferating cell nuclear antigen expression in endometrial carcinoma. Gynecol Oncol 1996;62:192-8.

26 Porter PL, Gown AM, Kramp SG, et al. Widespread p53 overexpression in human malignant tumours. Am f Pathol 1992;140:145-53.

27 Poremba C, Bankfalvi A, Dockhorn-Dworniczak B. Das tumoursuppressorgen p53. Pathologe 1996;17:181-8.

28 Berchuck A, Kohler MF, Mark JR, et al. The p53 tumour suppressor gene frequently is altered in gynecologic suppressor gene frequently is altered in gyne

29 Okamoto A, Sameshima Y, Yamada Y, et al. Allelic chromosome $17 \mathrm{p}$ and $\mathrm{p} 53$ mutations in human endometrial carcinoma of the uterus. Cancer Res 1992;52:6079-82.

30 Ambros RA, Vigna PA, Figge J, et al. Observations on tumour and metastatic suppressor gene status in endometrial carcinoma with particular emphasis on p53. Cancer 1994;73:1686-92.

31 Inoue $M$, Okayama A, Fujita $M$, et al. Clinicopathological characteristics of p53 overexpression in endometrial cancers. Int $\mathcal{F}$ Cancer 1994;58:14-19.

32 Kohler MF, Berchuck A, Davidoff AM, et al. Overexpression and mutation of p53 in endometrial carcinoma. Cancer Res 1992;52:1622-7.

33 Nazeer T, Curch K, Amato C, et al. Comparative quantitative immunohistochemical and immunoradiometric determinations of cathepsin D in endometrial adenocarcinoma: predictors of tumour aggressiveness. Mod Pathol 1994;7: 469-74.

34 Nazeer T, Malfetano JH, Rosano TG, et al. Correlation of tumour cytosol cathepsin D with differentiation and nvasiveness of endometrial adenocarcinoma. 7 Clin Pathol 1992;97:764-9.

35 Saffari B, Jones LA, el Naggar A, et al. Amplification and overexpression of HER-2/neu (c-erbB-2) in endometrial cancers: correlation with overall survival. Cancer Res 1995; 55:5693-8.

36 Wang D, Konishi J, Koshiyama M, et al. Expression of c-erbB-2 protein and epidermal growth factor receptor in c-erbB-2 protein and epidermal growth factor recept

37 Kohlberger P, Loesch A, Koelbl H, et al. Prognostic value of immunohistochemically detected HER-2/neu oncoprotein in endometrial cancer. Cancer Lett 1996;98:151-5.

38 Bigsby RM, Aixin L, Bomalaski J, et al. Immunohistochemical study of HER-2/neu, epidermal growth factor receptor, and steroid receptor expression in normal and malignant endometrium. Obstet Gynecol 1992;79:95-100. 